\title{
Transforming grief: models and integrative tools
}

\begin{abstract}
This article provides an overview of the grief process. An exploration of the various models of grief will be discussed. An exploration of integrative tools \& techniques designed to assist practitioners with identifying and treating potential grief $\&$ loss issues will be reviewed. A brief overview of the mind body connection \& the criteria proposed for traumatic grief diagnosis will be reviewed.

"Change is the only constant. But we are wired to hold on to what we have loved, to what was known and familiar. Therein lies much of our suffering since change always involves the loss of what was, making the way for what will be...."

Donna Eden and David Feinstein Co-Authors, The Energies of Love - excerpt from Peaks and Valleys: Integrative Approaches for Recovering from Loss by Sherry O'Brian.

Loss is unique because all individuals will eventually experience it in some form or other. Grief resulting from loss is often misunderstood and is prevalent among those suffering from chronic illness and other life experiences. Grief is both an emotion and a process. If grief is left untreated or unacknowledged, its effects can accumulate, causing psychological blocks that may interfere with one's ability to enjoy life.

Unfortunately, many individuals (and their counselors) are unaware they have experienced a loss. Therefore, they ignore or deny the emotional process of grieving, and the energy of these emotions becomes "frozen" within. As these emotions accumulate, they can cause each subsequent loss to be even more difficult to process. I believe one of the reasons this occurs is due to a misunderstanding of some available grief models, which I will briefly review below.
\end{abstract}

Volume 3 Issue I - 2016

Sherry O'Brian

Mind/Body/Spirit Inner-grations, USA

Correspondence: Sherry O'Brian, Mind/Body/Spirit Innergrations, 33 Metsker Lane Noblesville, IN 46062, USA, Tel 3174702328, Email slobrian247@sbcglobal.net

Received: October 09, 2015 | Published: January 29, 2016

\section{Models of the grief process}

\section{Stages of Grief model}

When I was first trained in bereavement counseling, the "stages of grief" model by Elizabeth Kubler Ross was used. The stages include: 1) denial, 2) anger, 3) bargaining, 4) depression, and 5) acceptance. The Elizabeth Kubler Ross model was developed while working with terminally-ill patients, and it was not originally meant to be adapted to the bereaved. However, at the time there were not many other grief models available to practitioners, so it was modified and used to assist individuals through the grief process. Unfortunately, it is still taught in various schools and hospice organizations as the standard bereavement model.

Although I find it useful sometimes, this model can confuse the bereaved because it implies that if we get through the various stages of grief, we can expect to finish the grief process. I believe this is one of the most misunderstood aspects of grief. Although we can learn to live with our losses, most of our losses are not forgotten, nor should they be.

\section{The four tasks of mourning model}

Another model that I believe is more appropriate for the bereaved is known as The Four Tasks of Mourning, which appears in Grief Counseling and Grief Therapy, Second Edition, by J William Worden.

These four tasks are:

1. Accepting the reality of the loss;

2. Working through the pain;

3. Adjusting to the environment; and
4. Emotionally relocating the deceased; that is, filing away in a safe place the mental and emotional memories of the deceased.

This model is specific to the loss of a loved one, but like the stages of grief model it implies a stage or step-like experience that we go through after a loss.

\section{The grief cycle model}

One of the models that I find most helpful when working with individuals who have experienced any kind of loss is referred to as the Grief Cycle. This cycle consists of shock, protest, disorganization, reorganization, and recovery. I want to emphasize that these are phases, not stages, which one moves through after a loss. Moreover, there is no time limit on grief, and although there are common issues that most individuals face after a loss, each of us will move through these phases at our own pace. The important thing to remember here is "movement." I believe that as long as my clients are continuing to move through these various phases they are recovering. The Grief Cycle can also be applied to other forms of loss such as relationships, dreams and youth. When I started to use this model, I began to notice how many of my patients were actually experiencing some form of grief/loss issue(s), or aspect(s) - regardless of their presenting problem.

\section{Integrative tools}

I began integrating various energy psychology tools to assist my clients in "releasing" each of the various aspects of grief presented. These aspects often included guilt, shame, fear, sadness, anger, etc. and several corresponding psychological reversals. When using these energy psychology tools, I noticed that several physical issues also improved, such as asthma and sinusitis - these conditions are often associated with grief/loss issues. For example, I had a client 
who complained of severe asthma. I used dx TFT, hypnotherapy, and subtle energy work with her. She was able to release the suppressed emotion of grief and her asthma cleared up after only three sessions.

I also began to notice that when trauma was involved with the grieving process some of my clients would disassociate. Energy psychology was one of the most effective tools to assist them in remaining present and discharging the traumatic energy associated with their experience. This is where my intuition and empathic sense of the client's energy/presence becomes crucial. For example, I often ask the client where they are, or where they went. I then help the client to pull back into the body using various tools such as heart massage, breath-work, and acupoint stimulation. EFT and the movie technique works well too, however, the client must be fully present to benefit.

\section{Using hypnosis and energy psychology to hea} ancestral grief

As I assisted individuals dealing with grief/loss issues, 1 became aware of another phenomenon I call "ancestral" grief" - "[whereas] whole communities can grieve as a result of group consciousness. The "morphic field" of groups, such as the American Indians, African Americans, the Jewish community, and so on, have endured mass trauma. This trauma is held at a cellular level within the human body and within the energetic field or group consciousness of these cultures. Other generations within these communities can continue to hold this energy of grief for generations to come unless it is recognized and released at a cellular level." (Peaks \& Valleys: Integrative Approaches For Recovering From Loss, by Sherry O’Brian).

For example, I had a client who struggled with depression. Upon questioning her, I realized that she was describing unresolved grief; however, she was unaware of any particular loss. Without going into all the details, I used hypnosis to help her find the source of her grief.
She went back to a time when her ancestors were being slaughtered. We resolved this at the source and freed her energetic connection to it using acupoint stimulation. I believe the study of epigenetics can also explain how these imprints on our genetic code may be passed down from generation to generation.

\section{Heart/brain coherence technique}

The heart is often involved with any type of loss. Establishing heart/brain coherence is very important for those recovering from this experience. To assist my clients I developed a technique called "holding the heart in mind," which is usually a quick and simple way to achieve heart/brain coherence. This involves holding the right hand over the heart chakra until a pulsation in the right hand is felt, then keeping the right hand over the heart, placing the left palm over the third eye (frontal lobe mid-forehead) until a pulsation in the left palm is felt, and then syncing (hooking up) the pulses. When the synchronization is complete lock it in by holding the hands in a prayer position in front of the heart chakra.

Using this technique and other integrative approaches such as various forms of energy psychology, hypnotherapy, guided imagery and incorporating the healing power of ritual in my work has been instrumental in assisting my clients in recovering from loss.

\section{Acknowledgments}

None.

\section{Conflicts of interest}

Author declares there are no conflicts of interest.

\section{Funding}

None. 